\title{
Transfer of Amino Acids across the In Vitro Perfused Human Placenta
}

\author{
H. SCHNEIDER, K.-H. MÖHLEN, AND J. DANCIS ${ }^{(22)}$
}

Department of Obstetrics and Gynecology, Department of Pediatrics, New York University School of Medicine, New York, New York, USA, Universitats-Frauenklinik, Marburg, and Universitats-Frauenklinik, Munster, West Germany

\section{Summary}

Amino acid transfer across human placenta has been studied in an in vitro perfusion system. Transfer rates from maternal to fetal circuit of 12 amino acids were about the same when there was a downhill gradient permitting diffusion and active transport. This was termed "maximal transfer rate." Cystine, aspartic acid, and glutamic acid were transferred at approximately half the rate of the others under these conditions. Measurement of exchange rates with radioactive tracers and with equimolar concentration of amino acids on both sides of the membrane provided a close estimate of maximal transfer rate. Fetal nutrition requires active transfer against a gradient. The rate of active transfer is a fraction of the exchange rate.

The ability to establish a transplacental gradient by perfused placenta was investigated with the maternal circuit open and fetal circuit closed (recirculated). The fetal to maternal concentration ratio for D-leucine and antipyrine was 1.0 , for L-leucine, 1.5 , indicating a stereospecific transport mechanism for leucine. Gradients of $1.56 \pm 0.11$ and $1.62 \pm 0.10$ were demonstrated for $L$ alanine and L-lysine, respectively. The establishment of transplacental gradients is a placental function, requiring no assistance from maternal or fetal factors.

The ratios of the exchange rates for the $L$ - and $D$-isomers were determined as a measure of the active, stereospecific transport system. The L:D ratio from the maternal to fetal circulation for leucine was $1.64 \pm 0.19$, for alanine $1.78 \pm 0.49$. In the reverse direction, the ratio for leucine was 1.03 . The placental membrane exhibits a polarity in active transport directed towards the fetus. The transplacental gradient for leucine is the result of a dynamic equilibrium between active transport towards the fetus and diffusion back towards the maternal circulation.

The availability of intracellular amino acids to serve as a reserve for fetal needs was studied by perfusing the placenta with buffer containing no amino acids. After $2 \mathrm{hr}$ of perfusion, 53-84\% of each of nine amino acids was still retained within the placenta. It is suggested that the high tissue concentrations of amino acids are related to the metabolic needs of the placenta rather than to its transport functions.

\section{Speculation}

The present studies have demonstrated differences between placental uptake and placental transfer of amino acids. It is still uncertain whether the two processes are serving two discrete functions (placental nutrition and fetal nutrition) or whether transplacental transfer involves mechanisms in addition to placental uptake (e.g., placental release).

Amino acids circulating in maternal plasma are the primary substrates for the synthesis of proteins by the fetus (6). Recent studies suggest that they may also contribute to the energy supply of the fetus (8). The mechanisms by which the amino acids are delivered by the placenta to the fetus have been extensively studied using a variety of experimental techniques and experimental animals. Studies in the human have been extremely limited. Slices and fragments of human placenta have been used in vitro to measure amino acid transport $(11,12,16,19)$, but the pertinence of the observations to transplacental transport is uncertain. Page et al. (13) have infused D- and L-histidine into several mothers shortly before delivery and have measured the relative concentrations of the stereoisomers in the cord blood at birth. They deduced that $\mathrm{L}$-histidine was transferred more rapidly than the D-isomer. Gaull et al. (7) have studied transfer rates of the sulfur-amino acids during the second trimester in elective abortions.

For the past several years, we have been studying transplacental transport by perfusing an isolated cotyledon of human placenta. Independent maternal and fetal circulations are established permitting the investigation of transfer in either direction. This technique has now been applied to the study of amino acid transport in an attempt to answer such fundamental questions as the relation of exchange rates to net transfer, the pertinence of in vitro placental uptake studies to transplacental transport, the polarity of the placental membrane, and the possible reserve functions of the large intraplacental concentrations of amino acids.

\section{MATERIALS AND METHODS}

\section{PERFUSION TECHNIQUE}

The technique of dual perfusion of the human placental cotyledon has been published in detail (17) and, therefore, only a brief description will be presented here. Fresh placentas obtained from uncomplicated term gestations by either vaginal or abdominal deliveries are inspected on both sides and a suitable cotyledon is identified. The artery and vein supplying the chorionic vascular system are dissected and fine polyethylene catheters are inserted and fixed by a 00 silk ligature. The perfusion of the fetal vascular tree is started immediately with Earle's buffered salt solution and the perfused portion is isolated from the rest of the placenta. The cotyledon is then turned upside down and fixed in a perfusion chamber filled with buffer solution. The decidual plate assumes a white color over the area which is perfused from the fetal side indicating an efficient rinsing of the fetal capillaries. Two fine glass cannulas are inserted into the intervillous space by penetrating the decidual plate over the light colored area and the intervillous space is perfused through these cannulas. The perfusion chamber, the two circuits and fluid reservoirs are mounted in a temperature controlled box at $37^{\circ}$. Samples can be taken separately from maternal and fetal inflow as well as outflow.

\section{ANALYTICAL METHODS}

Antipyrine. The radioactive antipyrine was separated from the radioactive amino acids by extracting $1 \mathrm{ml}$ of perfusate with $2 \mathrm{ml}$ chloroform three times. The $6 \mathrm{ml}$ of chloroform was evaporated to dryness under $\mathrm{N}_{2}$ in a scintillation vial and redissolved in 0.5 $\mathrm{ml} \mathrm{H}_{2} \mathrm{O}$. Diotol scintillant was added for determination of radioactivity. 
Amino Acids. Nonradioactive amino acids were analyzed with a Phoenix Microanalyzer (model PKU). The samples were deproteinized with picric acid (20). Two buffer systems were used: 0.2 $\mathrm{M} \mathrm{Na}^{+}, 0.05 \mathrm{M}$ citrate, titrated to $\mathrm{pH} 3.05$ (8) for the elution of the acid, neutral, and branched-chain amino acids and $0.35 \mathrm{~N}$ $\mathrm{Na}^{+}$buffer, at $\mathrm{pH} 4.68$ for the aromatic and basic amino acids (18). Lithium citrate buffer was used to achieve better separation of glutamic and aspartic acids and of their amides (2).

Radioactive amino acids were identified by paper chromatography. Five $\mathrm{ml}$ perfusate was mixed with an equal volume of $10 \%$ perchloric acid for deproteinization. After centrifugation, the $\mathrm{pH}$ of the supernatant was adjusted to 7.0 with $\mathrm{KOH}$ and again centrifuged. The supernatant was applied to Dowex $50\left(\mathrm{H}^{+}\right)$, the resin was washed with water, and the amino acids eluted with $2 \mathrm{~N}$ $\mathrm{NH}_{4} \mathrm{OH}$. The eluate was taken down to about $1 \mathrm{ml}$ in a Buchner flash evaporator and then blown to dryness in a small centrifuge tube, under $\mathrm{N}_{2}$ at $37^{\circ}$. The residue was dissolved in $50 \mu \mathrm{l}$ water and submitted to paper chromatography in butanol:acetic acid: water (12:3:5). Standard amino acids were chromatographed concurrently and located by development with ninhydrin. The parallel bands containing the radioactive amino acids were cut into small strips, and transferred to a scintillation vial and $3 \mathrm{ml}_{2} \mathrm{H}$ added and the amino acids were eluted by shaking overnight in an incubator at $35^{\circ}$. The strips were removed and the water evaporated under $\mathrm{N}_{2}$. The residue was redissolved in $0.5 \mathrm{ml} \mathrm{H}_{2} \mathrm{O}$ and Diotol added. Radioactivity was determined on a two-channel $\left({ }^{3} \mathrm{H}:{ }^{14} \mathrm{C}\right)$ scintillation counter.

Radioactivity in perfused tissue was analyzed in the same fashion after extracting $5 \mathrm{~g}$ of tissue with $5 \mathrm{ml}$ of $10 \%$ perchloric acid.

Nonlabeled amino acids were purchased from Schwartz-Mann, while radioactive amino acids and antipyrine (antipyrine- $\mathrm{N}-\left({ }^{3} \mathrm{H}\right)$ methyl) were obtained from New England Nuclear, except for ${ }^{14} \mathrm{C}$-D-glutamic acid which came from International Chemical and Nuclear Corp. All radioactive amino acids were uniformly labeled with either ${ }^{14} \mathrm{C}$ or ${ }^{3} \mathrm{H}$ and were tested for purity by paper chromatography.

\section{RESULTS}

The movement of amino acids across the perfused placental membrane is expressed as rates of transfer or rates of exchange. For the measurement of transfer rates, amino acids were added to only one perfusate, either maternal or fetal. The rate of transfer from maternal to fetal side was calculated from the amino acid concentration measured in the fetal vein and the flow-rate in the fetal perfusion circuit. In order to measure exchange rates, amino acids were added in equimolar concentration to both perfusates and radioactive labeled tracers were added to either maternal or fetal reservoir. The rate was calculated from the radioactivity measured in either maternal or fetal outflow and the respective flow-rate.

Transfer rates and exchange rates are generally presented as the percent of antipyrine transfer (clearance index or exchange index). The rationale for this has been previously discussed (4). In brief, by relating results to antipyrine transfer hemodynamic, anatomic, and technical variables are reduced permitting more reliable comparisons among experiments.

\section{MAXIMAL TRANSFER RATES (DIFFUSION PLUS ACTIVE TRANSPORT)}

Both maternal and fetal circulations were kept "open", that is, nor recirculated. Amino acids were added to the maternal perfusate in equimolar concentrations of $0.25 \mathrm{mM}$. No amino acids were added to the fetal perfusate, thereby establishing a transplacental gradient towards the fetal circulation. The experiment lasted for approximately $60 \mathrm{~min}$. Several $1 \mathrm{~min}$ samples were collected from the fetal outflow and the transfer rate was determined once steady state conditions had been achieved.

Table 1 lists the results expressed as the percent of antipyrine transferred, which was measured in each experiment. Of the 15 amino acids that were studied, all except three had similar transfer
Table 1. Transfer rates and exchange rates of amino acids

\begin{tabular}{|c|c|c|c|c|}
\hline & \multicolumn{2}{|c|}{ Clearance index' } & \multicolumn{2}{|c|}{ Exchange index ${ }^{2}$} \\
\hline & $\begin{array}{l}\text { No. of ex- } \\
\text { periments }\end{array}$ & Mean $\pm \mathrm{SE}$ & $\begin{array}{l}\text { No. of ex- } \\
\text { periments }\end{array}$ & Mean $\pm S E$ \\
\hline Aspartic acid & 5 & $16.7 \pm 2.8$ & 5 & $19.9 \pm 2.4$ \\
\hline Asparagine & 5 & $41.2 \pm 4.0$ & & \\
\hline Glutamic acid & 4 & $13.7 \pm 1.9$ & 4 & $19.2 \pm 3.1$ \\
\hline Glutamine & 5 & $38.5 \pm 4.5$ & 3 & $38.0 \pm 6.2$ \\
\hline Glycine & 7 & $35.2 \pm 4.1$ & & \\
\hline Alanine & 7 & $36.5 \pm 3.1$ & 3. & $33.7 \pm 3.8$ \\
\hline Cystine & 4 & $16.3 \pm 2.5$ & & \\
\hline Valine & 7 & $32.5 \pm 2.7$ & & \\
\hline Methionine & 5 & $34.6 \pm 3.1$ & & \\
\hline Leucine & 4 & $33.0 \pm 6.8$ & 7 & $36.0 \pm 3.11$ \\
\hline Tyrosine & 3 & $35.3 \pm 5.5$ & & \\
\hline Phe-alanine & 4 & $37.6 \pm 5.6$ & & \\
\hline Histidine & 4 & $34.5 \pm 2.8$ & & \\
\hline Lysine & 6 & $32.2 \pm 5.0$ & 3 & $27.8 \pm 3.6$ \\
\hline Tryptophan & 2 & $47.6(39.1-56.1)$ & & \\
\hline $\begin{array}{c}\alpha \text {-aminoisobu- } \\
\text { tyric acid }\end{array}$ & & & 8 & $27.5 \pm 2.3$ \\
\hline Arginine & & & 6 & $27.1 \pm 1.1$ \\
\hline
\end{tabular}

' Amino acids and antipyrine were added to maternal perfusate in equimolar concentrations $(0.25 \mathrm{mM})$. None was added to the fetal perfusate. The rates of transfer were determined during a $1 \mathrm{hr}$ perfusion. Results are expressed as clearance index.

$$
\text { C.I. }=\frac{\text { clearance of amino acid }}{\text { clearance of antipyrine }} \times 100
$$

Clearance $=\frac{[\mathrm{Fv}] \times \mathrm{Qf}}{[\mathrm{Ma}]} ;[\mathrm{Fv}]=$ concentration in fetal vein; $\mathrm{Qf}=$ fetal flow rate; $[\mathrm{Ma}]=$ concentration in maternal artery.

${ }^{2}$ Amino acids were added in equimolar concentrations to maternal and fetal perfusates. Trace amounts of radioactive amino acids and antipyrine were added to the maternal perfusate. Rates of exchange are expressed as exchange index.

$$
\text { Exchange index }=\frac{\text { exchange rate of amino acid }}{\text { clearance of antipyrine }} \times 100
$$

Exchange $=\frac{\left[\mathrm{Fv}^{*}\right] \times \mathrm{Qf}}{\left[\mathrm{Ma}^{*}\right]} ;\left[\mathrm{Fv}^{*}\right]=$ concentration of radioactive substrate in fetal vein; $\mathrm{Qf}=$ fetal flow rate; $\left[\mathrm{Ma}^{*}\right]=$ concentration of radioactive substrate in maternal artery. The clearance of antipyrine in 14 experiments was $1.99 \mathrm{ml} \pm 0.18$ (mean $\pm \mathrm{SEM}$ ). Clearance and exchange may be estimated from the indices by the utilization of this factor.

rates averaging about a third of antipyrine. Aspartic acid, glutamic acid, and cystine were transferred at approximately half the rate of the others.

\section{EXCHANGE RATES}

Equimolar concentrations of amino acids were added to the maternal and fetal perfusates, and trace amounts of radioactive amino acids and of radioactive antipyrine were added to the maternal perfusate. The rate of appearance of the radioactive amino acid in the fetal perfusate indicated the exchange rate. For the six amino acids that were studied by both techniques, the exchange index closely approximated the maximal transfer rate (clearance index) (Table IB).

To provide a more accurate comparison, exchange and clearance indices for two amino acids, L-leucine and L-aspartic acid, were measured together in three experiments (Table 2). In the first phase of the perfusion, exchange rate was measured with amino acids in both maternal and fetal perfusates. In the second phase, amino acids were omitted from the fetal perfusate and the transfer rates were again measured. The results were very similar, with exchange being slightly higher than transfer rate in each study. 


\section{TRANSPLACENTAL AMINO ACID CONCENTRATION GRADIENTS}

L-leucine- ${ }^{14} \mathrm{C}$, D-leucine- ${ }^{3} \mathrm{H}$, unlabeled $\mathrm{L}$ - and D-leucine, and antipyrine $-{ }^{3} \mathrm{H}$ were added in equal concentrations to the maternal and fetal perfusates. The maternal perfusate was not recirculated so that the concentration of the three constituents delivered to the placenta remained constant. The fetal circulation was closed. Samples were obtained at intervals for analysis.

Initially, the concentrations in the maternal and fetal circuits were the same (Fig. 1). The concentration in the fetal perfusates fell rapidly, as compared to the maternal perfusate because of dilution with contents of the fetal vascular system. Antipyrine rapidly equilibrated across the placenta reestablishing a fetal: maternal ratio of one. D-leucine also reequilibrated to equal concentrations, but at a slower rate. L-leucine, after the initial fall in fetal concentration, rose more rapidly than D-leucine and established a gradient of 1.5 .

Transplacental gradients of $1.56 \pm 0.11$ and $1.62 \pm 0.10$ were also established for L-alanine and L-lysine, respectively, in similar experiments.

\section{STEREOSPECIFIC EXCHANGE RATES}

L- and D-stereoisomers of leucine and alanine were added to maternal and fetal perfusates in equl concentration $(0.1 \mathrm{mM})$. Trace amounts of each isomer, differentially labeled with either ${ }^{3} \mathrm{H}$ or ${ }^{14} \mathrm{C}$, were added to the maternal perfusate. At the termination of the experiment, leucine and alanine were separated by paper

Table 2. Intraexperimental comparison of clearance and exchange index ${ }^{1}$

\begin{tabular}{|c|c|c|c|c|}
\hline \multirow[b]{2}{*}{. } & \multicolumn{2}{|c|}{ L-leucine } & \multicolumn{2}{|c|}{ L-aspartic acid } \\
\hline & $\begin{array}{c}\text { Exchange } \\
\text { index }\end{array}$ & $\begin{array}{c}\text { Clearance } \\
\text { index }\end{array}$ & $\begin{array}{c}\text { Exchange } \\
\text { index }\end{array}$ & $\begin{array}{c}\text { Clearance } \\
\text { index }\end{array}$ \\
\hline 1 & 53.0 & 47.2 & 26.0 & 23.9 \\
\hline 2 & 38.4 & 37.5 & 24.6 & 22.8 \\
\hline 3 & 36.4 & 30.0 & 14.3 & 14.1 \\
\hline Mean $\pm \mathrm{SE}$ & $42.6 \pm 5.2$ & $38.2 \pm 5.0$ & $21.6 \pm 3.7$ & $20.3 \pm 3.1$ \\
\hline
\end{tabular}

'Each perfusion consisted of two phases. The first phase measured exchange rate, with equimolar concentrations of amino acids in maternal and fetal perfusates. The second phase measured clearance index with amino acids in the maternal perfusate only. The clearance index was measured under conditions permitting maximal transfer rate (diffusion and active transport). See Table 1 for definition of terms.

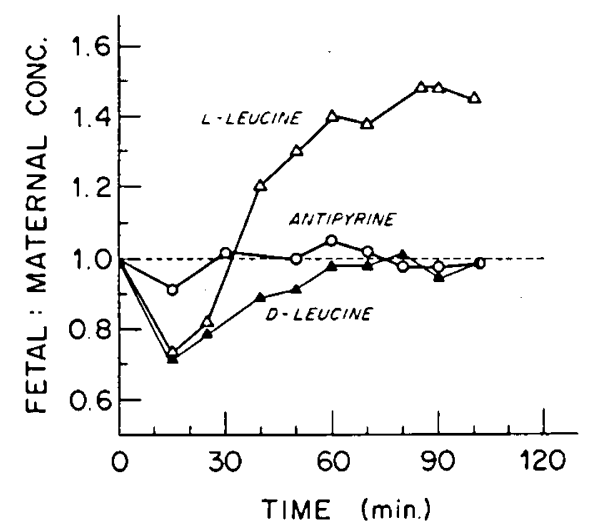

Fig. 1. Maternal and fetal perfusates initially contained equal concentrations of L- and D-leucine and antipyrine: Fetal:maternal concentration $=1$. Maternal perfusate was not recirculated so that concentrations remained unchanged. Fetal circuit was closed permitting fetal concentrations to seek equilibrium with maternal perfusate. The early decrease in ratio is caused by dilution with fluids retained within fetal vasculature. chromatography, the radioactive bands were eluted, and the ${ }^{3} \mathrm{H}:{ }^{14} \mathrm{C}$ ratios determined. The comparative exchange rates were calculated from the maternal:fetal ratios. The exchange rates for $\mathrm{L}$-leucine and L-alanine were greater than for their stereoisomers, providing average $L: D$ ratios of 1.64 and 1.78 , respectively (Table 3 ). In a similar study of exchange rates measured in the reverse direction, i.e., from the fetal perfusate, there was no difference between D- and L-leucine.

At the end of each perfusion, the perfused cotyledon was extracted and analyzed in the same way as the perfusates. The concentration of L-alanine in the tissue was twice as high as Dalanine, but there was no difference in the concentrations of the isomers of leucine.

\section{PLACENTAL CONTENT OF AMINO ACIDS}

After perfusion of placenta for $2 \mathrm{hr}$ with buffer containing no amino acids, the concentrations of amino acids in the perfused cotyledon were compared to those in an unperfused section of the placenta. In these studies, the greater proportion of amino acids was retained in the placenta (53-84\%) after $2 \mathrm{hr}$ of perfusion (Table 4).

A series of six experiments was performed and the rate of appearance of amino acids in maternal and fetal perfusates was measured. The results for alanine are presented in Figure 2. Similar results were obtained with glycine, taurine, aspartic acid, and glutamine. The concentration of amino acids eluted into the fetal perfusate falls rapidly during the first $30 \mathrm{~min}$ and then maintains a fairly constant rate for the rest of the experiment. Considerably more amino acid appears in the maternal perfusate and the rate of fall in concentration is more gradual.

\section{DISCUSSION}

Transfer characteristics of a series of 15 physiologic amino acids were investigated in the perfusion system. Transfer and exchange rates are expressed as percent of antipyrine transfer measured simultaneously (clearance or exchange index). By relating the amino acid results to antipyrine transfer, hemodynamic, anatomic, and technical variables are reduced permitting more reliable com-

Table 3. Relative exchange rates for $\mathrm{L}$ - and $\mathrm{D}$-isomers ${ }^{1}$

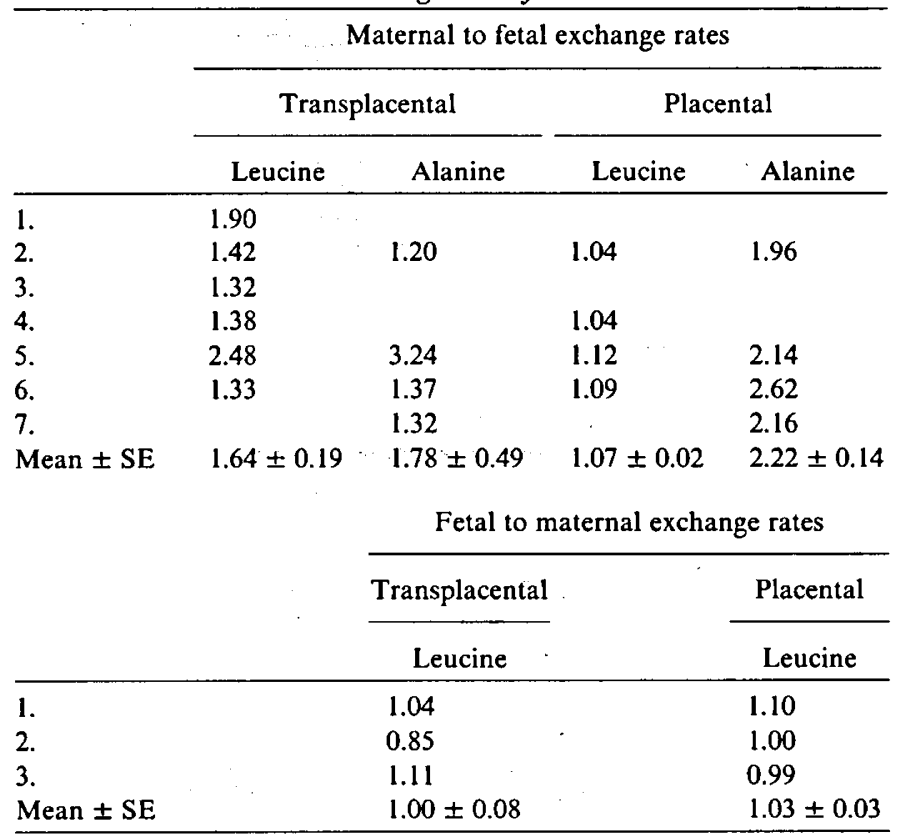

${ }^{1} \mathrm{~L}$ and $\mathrm{D}$ isomers, differentially labeled with either ${ }^{14} \mathrm{C}$ or ${ }^{3} \mathrm{H}$, were added to the maternal perfusate (A) or fetal perfusate (B). The ratios of their exchange rates ( $L: D$ isomers) into the placenta (placental exchange), and across the placenta (transplacental exchange) were determined. 
Table 4. Placental concentration of amino acids; release to perfusate.

\begin{tabular}{lccc}
\hline & $\begin{array}{c}\text { Unperfused } \\
\text { cotyledon } \\
(\mu \text { moles/g) }\end{array}$ & $\begin{array}{c}\text { Perfused } \\
\text { cotyledon } \\
(\mu \text { moles/g) }\end{array}$ & Retained (\%) \\
\hline Taurine & $3.61 \pm 0.05$ & $2.05 \pm 0.12$ &. \\
Aspartic acid & $2.16 \pm 0.05$ & $1.48 \pm 0.24$ & 66.8 \\
Asparagine & $0.64 \pm 0.09$ & $0.41 \pm 0.05$ & 64.5 \\
Glutamic acid & $3.78 \pm 0.45$ & $3.19 \pm 0.57$ & 84.4 \\
Glutamine & $0.79 \pm 0.10$ & $0.42 \pm 0.03$ & 53.2 \\
Glycine & $3.54 \pm 0.19$ & $2.70 \pm 0.19$ & 76.3 \\
Alanine & $3.16 \pm 0.19$ & $2.23 \pm 0.03$ & 70.6 \\
Threonine-serine & $4.39 \pm 0.19$ & $2.90 \pm 0.54$ & 66.1 \\
\hline
\end{tabular}

' Concentrations of amino acids in placenta were determined after 2 hours of perfusion of both maternal and fetal compartments with buffer containing no amino acids. Results are compared with an aliquot of the same placenta that had not been perfused. Mean $\pm \mathrm{SE}$ of six placentas.

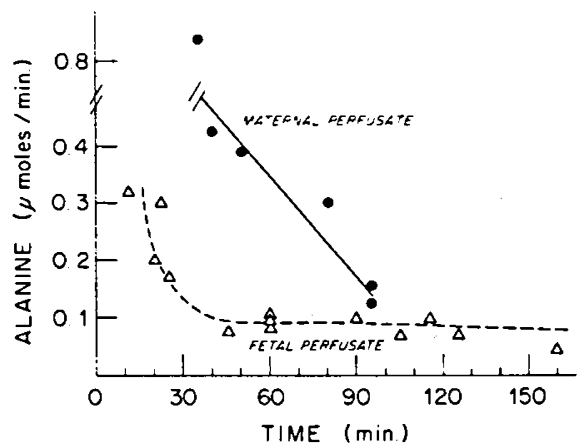

Fig. 2. Maternal and fetal compartments were perfused with buffer without amino acids. The recorded values were pooled from six perfusion experiments.

parisons among experiments (4): No-attempt has been made to translate the results into absolute transfer rates because the messages to be presented are dependent primarily on intra- and interexperimental comparisons.

Equimolar concentrations of amino acids were added to perfusates to permit a more accurate comparison of transfer rates. In the first series of experiments, a downhill gradient was established towards the fetal circulation by adding the amino acids to only the maternal perfusate. Under these conditions, it can be assumed that transfer will be by diffusion as well as by active transport mechanisms. We have termed this the maximal transfer rate. The transfer rates expressed as the "clearance index" of 12 of the 15 amino acids that were studied were about the same (Table 1). The three exceptions, aspartic acid, glutamic acid, and cystine were transferred at about half the rate of the others. From these observations, it is not possible to state whether the limitation in transfer of the three amino acids resulted from a reduction in diffusion rate, in active transport, or both. In vivo studies have shown a very slow transfer of cystine-cysteine across second trimester human placenta (7). Aspartic acid and glutamic acid will be discussed more fully in a later paper.

Exchange rates for six of the amino acids were also determined by adding radioactive tracers to the maternal perfusate with equimolar concentrations of amino acids in both maternal and fetal perfusates. The exchange indices approximated closely the results on transfer (Table 1 ) indicating that exchange rates measure transfer under conditions in which diffusion as well as active transport can occur. When exchange rate and the maximal transport rate were compared within alternate phases of each experiment, it could be demonstrated that the former is actually slightly higher (Table 2). Acceleration of transfer when substrate is present on both sides of the membrane has been observed for the transfer of amino acids through other membranes. It is consistent with the model of a carrier that "shuttles" back and forth across the membrane, more efficiently when carrying substrate in both directions.

Studies of transport across membranes performed in vivo with isotopic tracers are almost always measurements of exchange rates because the substance under study is generally present on both sides of the membrane. The usefulness of the information depends on the specifics of the physiologic and experimental situation. Diffusion rates of amino acids across the placenta is greater than the rates of active transport and, therefore, constitute a larger fraction of the exchange rate. However, placental transport towards the fetus in vivo is against a gradient eliminating diffusion as a mechanism. It is net transport that is the nutritionally significant figure and net transport is dependent solely on active transport.

To investigate whether the perfusion system is suitable to study the active transport process, the establishment of gradients with select amino acids was investigated. The fetal circuit was closed and the equilibration of $\mathrm{L}$-leucine, $\mathrm{D}$-leucine, and antipyrine across the placental membrane was studied. Antipyrine equilibrated rapidly attaining a maternal:fetal ratio of one, as expected. Dleucine also equilibrated at equal concentrations, at a slower rate L-leucine established a gradient (Fig. 1). Similar experiments with L-lysine and L-alanine also yielded the expected gradients toward the fetus.

These studies demonstrated that the perfusion system could be used to study active transport and that the establishment of transplacental gradients was a placental function that was not directly dependent on either maternal or fetal factors. It was also clear that active transport was stereospecific for the L-amino acid and that the D-isomer was transferred solely or primarily by diffusion processes. It then followed, logically, that the ratio of the transfer or exchange rates of the L-amino acid to D-amino acid, would provide a measure of active transport.

The reasoning is the same as that used by Page et al. (13) in their study of placental transfer of histidine in vivo. These investigators infused $\mathrm{D}$ - and $\mathrm{L}$-histidine into a series of pregnant women at varying intervals before delivery in amounts that were sufficient to raise the maternal concentration of each isomer to approximately $3-5 \mathrm{mg} \%$. The relative concentrations of the isomers were then determined in cord blood at the time of birth. L-histidine equilibrated across the placenta more rapidly than D-histidine indicating a more rapid rate of transfer. The perfusion technique offered several technical advantages over this approach. The stereoisomers could be differentially labeled with radioactive isotopes simplifying their identification and increasing the accuracy of the assays; the complicating features of maternal and fetal metabolism were eliminated; the circulations could be kept "open", i.e., the perfusates were not recirculated permitting multiple sampling with constant levels of the amino acids on both sides of the placenta so that relative transfer rates could be accurately determined; exchange rates in both directions across the placenta were measured.

A series of studies was undertaken comparing the exchange rates of the stereoisomers of leucine and alanine (Table 3). The ratios for the exchange rates towards the fetal circulation averaged 1.64 and 1.78, respectively. Similar studies in the reverse direction demonstrated no difference in exchange rates between $D$ - and $L$ leucine. Active transport across the placenta appears to be unidirectional, towards the fetus. Transfer in the reverse direction occurs only by diffusion. Establishment of transplacental gradients for amino acids appears to be dependent on a dynamic equilibrium in which active transport towards the fetus is counterbalanced by diffusion pressure in the reverse direction. We have demonstrated comparable, though not as clearly interpretable, results using the in situ perfusion techniques of placenta in the guinea pig (5).

Amino acid uptake by placental slices and fragments has been studied in this laboratory (16) and in others $(11,12,19)$. Compar- 
ison of our results on transfer into the placenta with slices and the results on transfer through the placenta using the perfusion technique does not reveal any simple relation. Alanine is taken up from the incubation medium relatively rapidly by slices, developing an 8-fold intracellular gradient in an hour. In contrast, the intracellular concentration of leucine remained below that of the incubation medium because of a very rapid efflux rate (16). In the present perfusion studies, alanine is also concentrated within the placenta more effectively than leucine (Table 3). Despite these striking differences, the transfer rates and the transplacental gradients for the two amino acids were about the same.

We have suggested, based on observations in the guinea pig (5), that placental transfer of amino acids across the placenta might involve an active uptake from the maternal circulation, concentration of the amino acids within the placenta, followed by passive release down a concentration gradient into the fetal circulation. The present observations suggest that the mechanism for transplacental transfer must be at least partly independent of the intraplacental concentration process to accommodate the major differences between alanine and leucine.

It has been speculated that the high concentration of amino acids in placenta may serve as a reserve, reducing the effect of changes in maternal serum levels on circulating levels in the fetus. The availability of placental amino acids for this purpose was investigated by perfusing placenta with buffer containing no amino acids. There was an immediate release of amino acids into the maternal and fetal perfusates (Fig. 2). The rate of release and the amount released into the maternal circulation was greater than into the fetal circulation possibly because of the anatomic proximity to trophoblast. There were two phases to the rate at which amino acids appeared in the fetal perfusate. During the first 30 min, there was a rapidly falling rate followed by a slow sustained release for the remainder of the experiment. At the end of $2 \mathrm{hr}$ perfusion, the greater proportion of the amino acids (53-84\%) was still retained within the placenta (Table 4). The intracellular retention was, in fact, even greater than these figures imply because no correction was made for dilution by residual perfusate in intravascular and interstitial fluids. One must conclude that under the conditions of those studies the majority of placental amino acids are not freely available to the fetus.

It is more likely that the intracellular concentrations of amino acids are more closely related to its intrinsic metabolic requirements than to its transport functions. The placenta is an active metabolic organ with significant oxygen requirements (3) and involved in the synthesis of large amounts of protein. Similar amino acid concentrations have been reported in other active metabolic organs such as liver, brain, and muscle $(1,14,15)$.

\section{REFERENCES AND NOTES}

1. Battistin, L.: The uptake of various amino acids by the mouse brain in vivo. Brain Res., 29: 85 (1971).

2. Benson, J. K., Gordon, M. J., and Patterson, J. A.: Accelerated chromatographic analysis of amino acids in physiological fluids containing glutamine and asparagine. Anal. Biochem., 18: 228 (1967).

3. Challier-J. -C., Schneider, H., and Dancis, J.: In vitro perfusion of human placenta. V. Oxygen consumption. Am. J. Obstet. Gynec., 126: 261 (1976).

4. Dancis, J., Jansen, V., Kayden, H. J., Schneider, H., and Levitz, M.: Transfer across perfused human placenta. II. Free fatty acids. Pediatr. Res., 7: 192 (1973).

5. Dancis, J., Money, W. L.. Springer, D., and Levitz. M.: Transport of amino acids by placenta. Am. J. Obstet. Gynec., 101: 820 (1968).

6. Dancis, J., and Shafran, M.: The origin of plasma proteins in the guinea pig fetus. J. Clin. Invest., 37: 1093 (1958).

7. Gaull, G. E., Raiha, N. C. R., Saarikoski, S., and Strum, J. A.: Transfer of cyst(e)ine and methionine across the human placenta. Pediatr. Res., 7: 908 (1973).

8. Gresham, E. L., James, E. J., Raye, J. R., Battaglia, F. C., Markowski, E. L., and Meschia, G.: Production and excretion of urea by the fetal lamb. Pediatrics, 50: 372 (1972).

9. Hamilton, P. B.: Ion exchange chromatography of amino acids. A single column, high resolving, fully automatic procedure. Anal. Chem., 35: 2055 (1963).

10. Hill, M. M., and Young. M.: Net placental transfer of free amino acids against varying concentrations. J. Physiol.. 235: 409 (1973).

11. Longo, L. D., Yuen, P.; and Gussek, D. J.: Anaerobic glycogen-dependent transport of amino acids by the placenta. Nature, 243: 531 (1973).

12. Miller, R. K., and Berndt, W. O.: Characterization of neutral amino acids accumulation by human term placental slices. Am. J. Physiol., 227: 1236 (1974).

13. Page, E. W., Glendenning, M. B., Margolis, A., and Harper, H. A.: Transfer of D- and L-histidine across the human placenta. Am. J. Obstet. Gynec., 73: 589 (1957).

14. Ryan, W. L.: Immediate and prolonged effects of hydrocortisone on the free amino acids of rat skeletal muscle. Soc. Exp. Biol. Med. Proc., 114: 816 (1963).

15. Ryan, W. L., and Corner, M. J.: Free amino acids of human fetal and adult liver. Nature, 212: 292 (1966).

16. Schneider, H., and Dancis, J.: Amino acid transport in human placental slices. Am. J. Obstet. Gynec., 120: 1092 (1974).

17. Schneider, H., Panigel, M., and Dancis, J.: Transfer across the perfused human placenta of antipyrine, sodium and leucine. Am. J. Obstet. Gynec., 114: 822 (1972).

18. Seely, J. H., Edattel, S. R., and Benoiton. N. L.: A quick separation of the $\epsilon-N-$ methyl lysines using the amino acid analyzer. J. Chromatogr., 44: 618 (1969).

19. Smith, C. H., Adcock, E. W., Teasdale, F., Meschia, G., and Battaglia, F. C.: Placental amino acid uptake: tissue preparation, kinetics and pre-incubation effect. Am. J. Physiol., 224: 558 (1973).

20. Stein, W. H., and Moore, S.: The free amino acids of human blood plasma. J. Biol. Chem., 211: 915 (1954).

21. This work was presented, in part, at the 24th Annual Meeting of the Society for Gynecological Investigation, Tuscon, AZ, March 23-25, 1977.

22. This research was supported by National Institutes of Health Grant HD 00462.

23. Requests for reprints should be addressed to: Dr. Joseph Dancis, Department of Pediatrics, NYU Medical Center, 550 First Avenue, New York, New York 10016 (USA).

24. Received for publication January 6, 1978.

25. Accepted for publication May 3, 1978. 